\title{
A Low-Cost High Performance GaAs MMIC package using Air-Cavity Ceramic Quad Flat Non-Leaded Package up to $40 \mathrm{GHz}$
}

\author{
Young-Ho Suh, David Richardson, Anna Dadello, Simon Mahon, and James T. Harvey \\ Mimix Broadband Inc., 10795 Rockley Rd, Houston, TX 77099, USA \\ E-mail: ysuh@mimixbroadband.com, Phone: 1-281-988-4600
}

\begin{abstract}
A novel low-cost QFN (Quad Flat NonLeaded) package is introduced for GaAs MMIC packaging. The air-cavity QFN package is designed and implemented with an HTCC process using Alumina substrate. To operate the package up to $40 \mathrm{GHz}$, several key methods are proposed in this paper. A low noise and a buffer amplifier are packaged for practical application examples and compared with bare die measurements to verify the QFN package performance. A maximum of $4.5 \mathrm{~dB}$ insertion loss degradation occurred at $40 \mathrm{GHz}$. To authors' knowledge, this is the highest operating frequency for QFN type packages with low loss ever reported.
\end{abstract}

\section{INTRODUCTION}

Recently, several research activities have been reported for MMIC packages. Ceramic-based packages were introduced in [1] and [2] with high RF performances but required relatively high manufacturing costs. Plastic packages can reduce the material cost but have large fluctuation in CTE (Coefficient of Thermal Expansion), which induces fabrication inaccuracy. This kind of inaccuracy gives poor production yields as well as performance deterioration for the package. Organicbased packages were also introduced in 2004 [3]-[6]. Rogers 4003C/4350B materials were used for package construction in [3]-[6]. Organic-based packages have cheaper material cost and its relatively low dielectric constant enables extended operating frequency as reported in [7]. However, the general design rules of organic material processes limits high precision layer-tolayer registration for multi-layer package structure, and filled via sizes/locations are also limited due to the process nature. Organic-based packages reported in [3][5] operate up to $\mathrm{Ku}$-band. Heijningen and Priday reported the organic-based package can be used up to 40 $\mathrm{GHz}$ in 2004 [6], but the structure was fragile at the bottom layer due to thin copper plating inside a cavity. The organic-based package also has poor CTE, which reduces production yield together with RF performance deterioration. The plastic or organic-based packages also have outgas or erosive gas problems.

QFN (Quad Flat Non-leaded) packages are very popular in industry because of their low cost and easy availability. QFN packages usually have been used in lower frequency such as L, S or C band with relatively low power. Recently, Bessemoulin et al. succeeded in packaging and operating $\mathrm{Ku}$-band MMIC with air-cavity
QFN [8] with around 2-3 dB gain deterioration compared to the bare die.

In this paper, a very low-cost air-cavity ceramic QFN package operating up to $40 \mathrm{GHz}$ is introduced. Design rules and methods to extend operation up to $40 \mathrm{GHz}$ are described. Material cost for the package including ceramic lid is less than $\$ 1.00$ per piece at 100,000 piece quantities. Since it uses ceramic material, it has low CTE, high layer-to-layer registration precision due to HTCC process, and it has no outgas or erosive gas problems, all of which reduce device's performance and lifetime. CST Microwave Studio [9] was used to perform 3D electromagnetic simulation, which uses FDTD algorithm for full wave electromagnetic simulations.

\section{PACKAGe DeSign}

The structure of $5 \mathrm{~mm} \times 5 \mathrm{~mm}$, air-filled QFN package is illustrated in Fig. 1. The package consists of two layers made of Alumina $\left(\mathrm{Al}_{2} \mathrm{O}_{3}\right)$ substrates with a dielectric constant of 9.8. The substrate thicknesses for layer 1 and 2 are $0.008(200 \mu \mathrm{m})$ and 0.004 inches $(100 \mu \mathrm{m})$, respectively. Two layers are co-fired using HTCC process. Since layer 1 needs to be laser drilled for filled via holes, it easily can lose its flatness during high temperature HTCC process if a thin substrate is used. If the thickness of layer 1 is too thick, it will have long RF vertical vias, which will increase parasitic inductance. The thick layer 1 substrate also increases parasitic parallel plate mode capacitance between top and bottom ground pads of layer 1, which limits RF performance of the package.

A method to minimize this parasitic capacitance will be discussed in detail later is this article. Another important issue is thermal resistance of the package. A thicker substrate will not be desirable for good thermal conductivity for power devices, affecting device's performance and lifetime. Therefore, it is important to choose proper thickness of layer 1.

One of the main limits for a QFN type package's operating frequency is long bonding wire/ribbon length. This creates large inductance at high frequencies, which causes significant impedance mismatches. The thickness of layer 2 is chosen to have an identical height to that of the GaAs MMIC substrate to minimize bonding wire/ribbon length. 


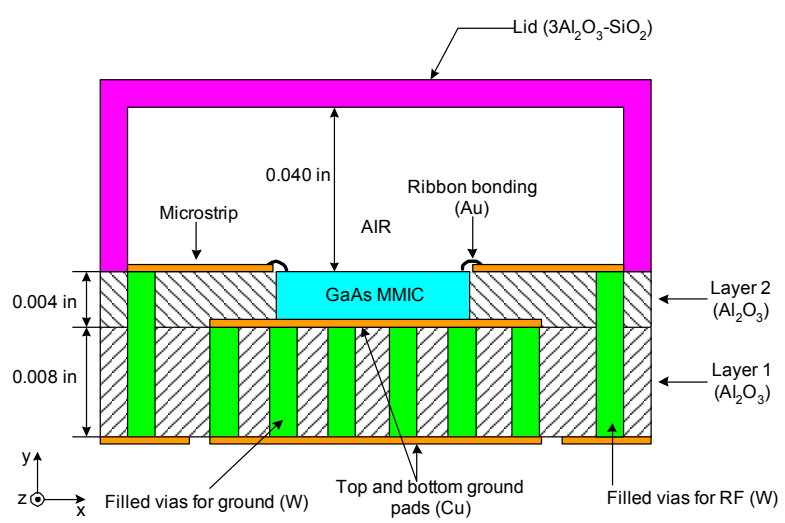

Fig. 1 . The structure of $5 \times 5 \mathrm{~mm}^{2}$, air-cavity ceramic QFN package.

Layer 2 also has a cutout to accommodate a MMIC and single layer DC decoupling chip capacitors, and a $50 \Omega$ microstrip is etched from RF vias to cutout edges to achieve short wire/ribbon RF connections between the MMIC and the microstrip line on layer 2. The cutout on layer 2 is shown in Fig. 2. In this work, 99.99\% gold bonding ribbon with 0.003 inch width and 0.001 inch height is used since it has lower inductance than that of bonding wire. Another benefit of this structure is only the cutout and RF/DC line patterns of layer 2 need to be replaced to accommodate different dimensions of other MMICs while layer 1 remains the same as shown in Fig. 2.

RF/DC and ground vias are filled with Tungsten, which has good thermal conductivity. Filled vias play important roles in both electrical grounding and thermal heatsinking of the package from top to bottom layer. The diameter for all vias is chosen to be around 0.008 inches.

Thermal simulation was performed using HEAT3 software [10] for three-dimensional transient and steadystate heat conduction analysis. The thermal conductivities of Alumina substrate and Tungsten filled via are 17 and $26(\mathrm{~W} / \mathrm{m} \cdot \mathrm{K})$, respectively. The simulation included RO4003C evaluation board with gold plated thru via holes for realistic conditions. The thermal conductivities of RO4003C board and gold plated thru via holes are about 0.64 and $21.4(\mathrm{~W} / \mathrm{m} \cdot \mathrm{K})$, respectively. Gold plated thru via holes are aligned with Tungsten filled vias of the package for good thermal conduction. A back plate temperature was assumed about $85^{\circ} \mathrm{C}$. With these conditions, simulation results show that temperature raise due to the package is about $11^{\circ} \mathrm{C}$ and $23^{\circ} \mathrm{C}$ with $0.5 \mathrm{~W}$ and $1 \mathrm{~W}$ MMIC dissipated power, respectively. The thermal simulation results show that the package is useful for low or medium power MMICs with moderate temperature raise.

Via pitch along $\mathrm{x}$-axis is designed around 0.026 inches. Via pitch along $\mathrm{Z}$-axis is designed to have 0.021 inches for $1^{\text {st }}$ to $3^{\text {rd }}$ row, 0.030 inches for $3^{\text {rd }}$ to $4^{\text {th }}$ row, and 0.031 inches for $4^{\text {th }}$ to $6^{\text {th }}$ row. It is important to place the $3^{\text {rd }}$ and $4^{\text {th }}$ rows of ground vias close to microstrip-to-RF vertical transition area because electric fields are easily scattered near this area causing a radiation loss as well as unwanted resonances [11]. The pitch between the $3^{\text {rd }}$ and $4^{\text {th }}$ row is 0.030 inches and it has been optimized with CST Microwave Studio. One notable thing is vias near edges of top/bottom ground pad are flush with borders of top and bottom ground pads on layer 1 . This has been done because ground pads on top and bottom of layer 1 can induce parallel plate mode creating fringing capacitances at high frequencies, causing RF performance degradation. Parasitic wave propagation also occurs along the borders of ground pads if the distance between edge vias and ground pad borders is wide [7]. Therefore, this condition should be included as one of the design conditions for high frequency QFN packages.

Electric field distributions of the package are illustrated in Fig. 3. RF via location also affects $R F$ performance. As shown in the circled area in Fig 3, part of electric fields is diverted in the top package's dielectric due to perturbation with the package dielectric to microstrip of RO4003C at the interface area. This kind of dielectric perturbation induces insertion loss and phase delay [12].
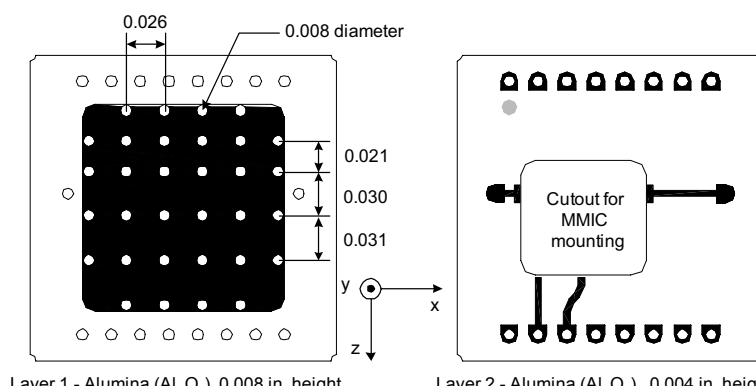

Layer 1 - Alumina $\left(\mathrm{Al}_{2} \mathrm{O}_{3}\right), 0.008$ in. height

Layer 2 - Alumina $\left(\mathrm{Al}_{2} \mathrm{O}_{3}\right),, 0.004$ in. height

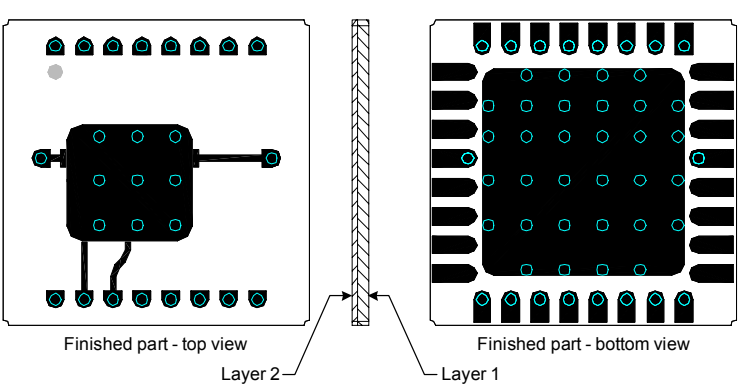

Fig. 2. Ceramic QFN package with two layers and filled vias (top view).

If the RF vias are located far inside the package, the length of perturbed microstrip on top of RO4003C would be long. This will cause severe insertion loss deterioration with large phase delay especially in $\mathrm{Ka}$ band [12]. For the same reason, the lid should not cover RF vias to prevent electric field from scattering to the lid area. Therefore, the RF vias need to be positioned as close as possible to the border of QFN package. The center of RF vias is located about 0.026 inches from the border of package.

The package needs to have an air-cavity structure for low insertion loss microwave and millimeter-wave applications. The dielectric constant of ceramic lid determines the spurious dielectric resonance in the millimeter-wave range. Low dielectric constant material 
gives a higher operating cut-off frequency of the package. For a ceramic lid material, Mullite $\left(3 \mathrm{Al}_{2} \mathrm{O}_{3}-\right.$ $\mathrm{SiO}_{2}$ ) was chosen, which has comparably low dielectric constant of 6.0 .

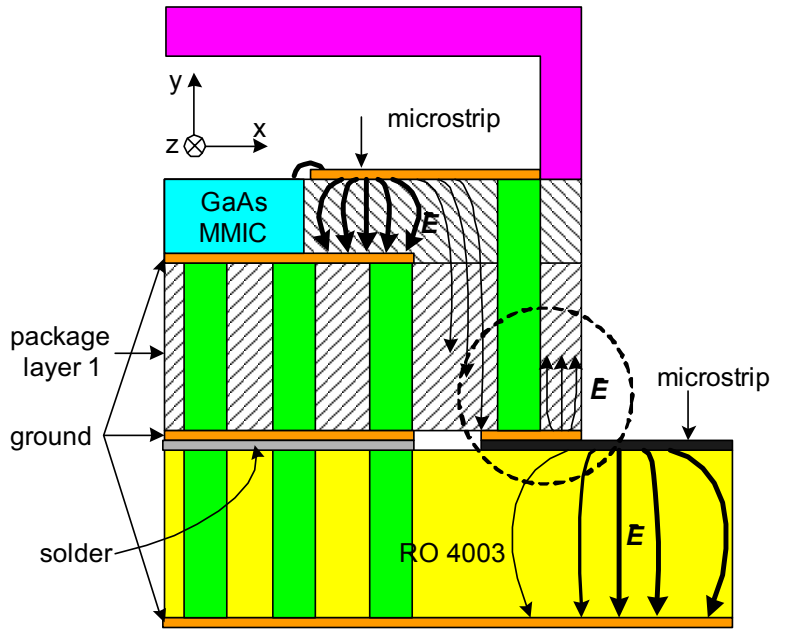

Fig. 3. Electric field distribution near RF vertical via of the package.

\section{GaAs PHEMT MMIC DESIGNS}

\section{A. Low Noise Amplifier}

XL1000 (Mimix Broadband) is an unbalanced, threestage, self-biased low-noise amplifier operating from 20 to $40 \mathrm{GHz}$. Small signal gain is about $20 \mathrm{~dB}$ from 20 to $30 \mathrm{GHz}$ and $16 \mathrm{~dB}$ from 30 to $40 \mathrm{GHz}$. Noise figure is around $3.0 \mathrm{~dB}$ from 20 to $30 \mathrm{GHz}$ and $3.8 \mathrm{~dB}$ from 30 to $40 \mathrm{GHz}$. It has been designed with Mimix Broadband's $0.15 \mu \mathrm{m}$ gate length GaAs pHEMT device technology. LNAs designed in this technology exhibit lowest noise at $\mathrm{V}_{\mathrm{gs}}=-0.3 \mathrm{~V}$, and can also be biased at $\mathrm{I}_{\mathrm{dss}}$ with $\mathrm{V}_{\mathrm{gs}}=$ $0.0 \mathrm{~V}$ to deliver 2 to $3 \mathrm{~dB}$ more output power and about $1 \mathrm{~dB}$ per stage increased gain. The first stage has total of $100 \mu \mathrm{m}$ gate width. Second and third stages have total of $150 \mu \mathrm{m}$ gate width each. The device was fabricated on $100 \mu \mathrm{m}$ thick GaAs substrate and the final MMIC has dimensions of $2 \mathrm{~mm} \times 1 \mathrm{~mm}$.

Each stage is configured to be self-biased at the low noise bias point, removing the need for a negative gate voltage for FET biasing. The device requires single $+5 \mathrm{~V}$ supply for all thee stages in parallel, and draws about 50 $\mathrm{mA}$ total drain current. The MMIC is unconditionally stable.

\section{B. Buffer Amplifier}

XB1004 (Mimix Broadband) is a single-ended, threestage buffer amplifier operating from 16 to $29 \mathrm{GHz}$. XB1004 offers two types of DC bias schemes. Two different types of biasing enable the device to operate in either low noise or buffer amplifier mode. Low noise amplifier mode can be operated at single $+4.0 \mathrm{~V}$ supply for all stages in parallel with $90 \mathrm{~mA}$ total drain current. This bias condition yields around $3.0 \mathrm{~dB}$ noise figure from 16 to $29 \mathrm{GHz}$. The small signal gain with this bias is about $+20 \mathrm{~dB}$. The output $1 \mathrm{~dB}$ compression point is about $+15 \mathrm{dBm}$ across the band. The buffer amplifier mode is operated at single $+6.0 \mathrm{~V}$ supply for all stages in parallel with $180 \mathrm{~mA}$ total drain current. The small signal gain becomes about $+23 \mathrm{~dB}$, which is around $3 \mathrm{~dB}$ higher than low noise amplifier mode. The output $1 \mathrm{~dB}$ compression point is also about $+18 \mathrm{dBm}$, which is a suitable output power level for driving LO for passive mixers or other high power amplifiers. In this work, the buffer amplifier mode is used for the package test.

It also has been designed with Mimix Broadband's $0.15 \mu \mathrm{m}$ gate length GaAs pHEMT device technology. The first and the second stages have a total of $200 \mu \mathrm{m}$ gate width each. The third stage has a total of $400 \mu \mathrm{m}$ gate width. The device was fabricated on $100 \mu \mathrm{m}$ thick GaAs substrate and final MMIC has dimensions of 2.33 $\mathrm{mm} \times 1.5 \mathrm{~mm}$.

\section{MEASUREMENTS}

The picture of packaged XL1000 is shown in Fig. 4. A single layer chip capacitor with capacitance of $100 \mathrm{pF}$ is included inside the package for DC decoupling. The evaluation board was built with 0.008 inch height RO4003C substrate for mounting the packaged MMIC. The evaluation board was tested with a Wiltron universal test fixture. The measurements were de-embedded with RO4003C microstrip line and universal test fixture loss. The comparison between die and package is shown in Fig. 5. As shown in Fig. 5, maximum gain deviation between die and packaged MMIC is around $4.5 \mathrm{~dB}$ near $40 \mathrm{GHz}$.

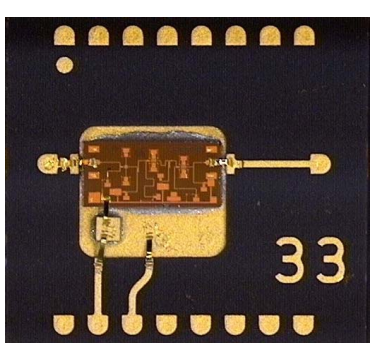

(a)

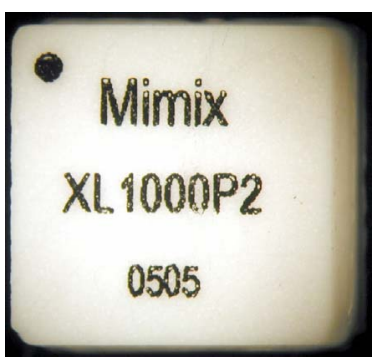

(b)
Fig. 4. Packaged XL1000 MMIC on air-cavity ceramic QFN package; (a) without lid, (b) with ceramic lid.

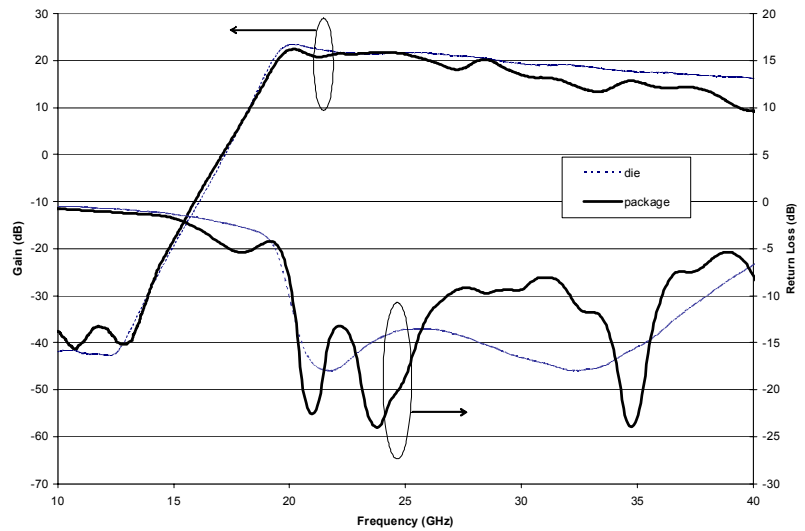


Fig. 5. Performance comparison between bare die and packaged XL1000 MMIC.

Maximum return loss of $7 \mathrm{~dB}$ was achieved across the band.

The picture of packaged XB1004 MMIC is also shown in Fig.6. Two 1nF single layer chip capacitors are used for DC decoupling for drain and gate. Maximum $3.5 \mathrm{~dB}$ gain deviation occurred at $29 \mathrm{GHz}$ and return loss is better than $9 \mathrm{~dB}$ across the band. The average gain of 21 $\mathrm{dB}$ was achieved from 16 to $29 \mathrm{GHz}$. Small balanced tuning stubs were placed at the end of microstrip on Alumina, where the bonding ribbons are joining. This improves the insertion and return loss which are normally degraded by bonding ribbon's inductance.

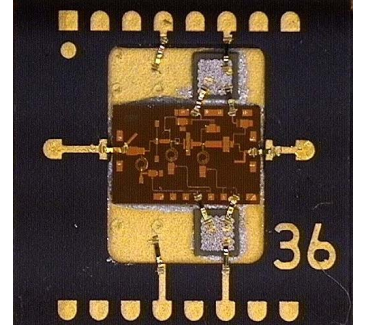

(a)

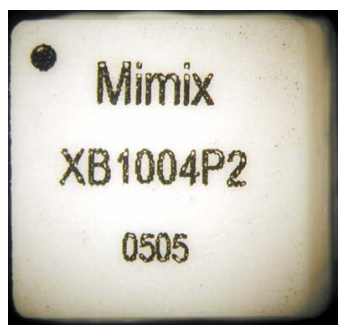

(b)
Fig 6. Packaged XB1004 MMIC on air-cavity ceramic QFN package; (a) without lid, (b) with ceramic lid.

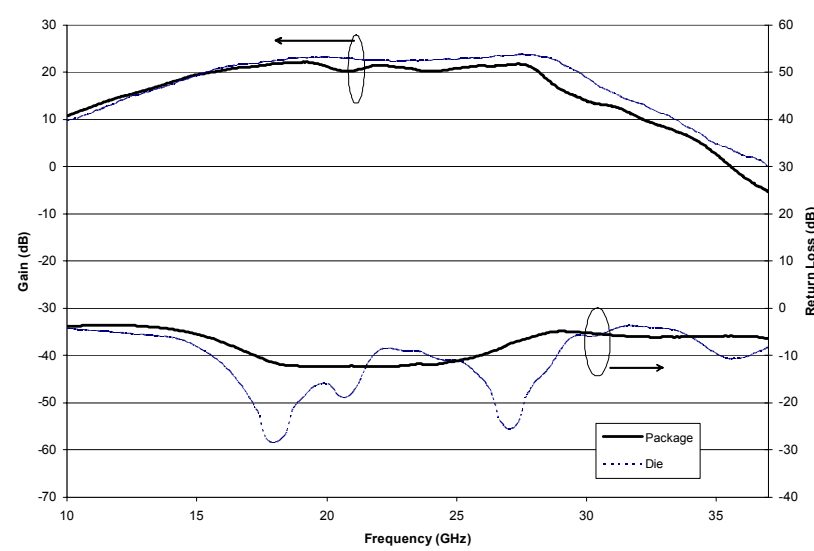

Fig. 7. Performance comparison between bare die and packaged XB1004 MMIC.

\section{CONCLUSION}

A ceramic based air-cavity QFN packages were designed and tested with two Ka-band MMICs. The design conditions for high frequency packaging were described and discussed. The material thickness of each layer was chosen to have good thermal conductivity, flatness, and low vertical via inductance. Ground via locations are optimized to enhance maximum operating frequency. The packaged MMICs have around 3 and 4.5 $\mathrm{dB}$ gain degradations at 29 and $40 \mathrm{GHz}$ for XB1004 and XL1000, respectively. The developed package will find useful applications for high frequency MMICs with remarkably low cost.

\section{ACKNOWLEDGEMENT}

The authors wish to acknowledge Justin Goodman in NTK Technology at Richardson, TX USA for technical discussion. The authors also would like to thank Thomson Corporate Research in Rennes, France for technical collaboration especially in thermal simulations.

\section{REFERENCES}

[1] S. Koriyama, K. Kitazawa, N. Shino, and H. Minamiue, "Millimeter-wave ceramic package for a surface mount," IEEE MTT-S Int. Microwave Symposium Digest, pp. 6164, Boston, MA, June 2000.

[2] H. C. Huang, A. Ezzeddine, A. Darwish, B. Hsu, J. Williams, and S. Peak, "Ku-band MMIC's in low-cost, SMT compatible packages," IEEE MTT-S Int. Microwave Symposium Digest, pp. 27-30, Seattle, WA, June 2002.

[3] A. Bessemoulin, M. Parisot, P. Quentin, C. Saboureau, M. van Heijningen, and J. Priday, "A 1-watt Ku-band power amplifier MMIC using cost-effective organic SMD package," in Proceedings of the $34^{\text {th }}$ European Microwave Conference, Amsterdam, Netherlands, October, 2004, pp. 349-352.

[4] K. Fujii, H. Morkner, and E. Brown, "A novel low cost enhancement mode power amplifier MMIC in MST package for 7 to $18 \mathrm{GHz}$ applications," in Proceedings of the $34^{\text {th }}$ European Microwave Conference, Amsterdam, Netherlands, October, 2004, pp. 361-364.

[5] A. Bessemoulin, M. Parisot, P. Quentin, C. Saboureau, M. van Heijningen, and J. Priday, "A 1-watt Ku-band power amplifier MMIC using cost-effective organic SMD package," in Proceedings of the $34^{\text {th }}$ European Microwave Conference, Amsterdam, Netherlands, October, 2004, pp. 349-352.

[6] M. van Heijningen, and J. Priday, "Novel organic SMD package for high-power millimeter wave MMICs," in Proceedings of the $34^{\text {th }}$ European Microwave Conference, Amsterdam, Netherlands, October, 2004, pp. 357-360.

[7] T. Tischler, M. Rudolph, A. Kilk, and W. Heinrich, "Via arrays for grounding in multilayer packaging - frequency limits and design rules," IEEE MTT-S Int. Microwave Symposium Digest, pp. 1147-1150, Philadelphia, PA, June 2003.

[8] A. Bessemoulin, M. Parisot, and M. Camiade, "1-watt Kuband power amplifier MMICs using low-cost quad-flat plastic package," IEEE MTT-S Int. Microwave Symposium Digest, pp. 473-476, Fort Worth, TX, June 2004

[9] "CST Microwave Studio" version 5.1, CST Computer Simulation Technology Inc., 2004.

[10] T. Blomberg, "HEAT3" version 3.0, Department of Building Physics, Lund University, Lund, Sweden, 2001.

[11] M. Ito, K. Maruhashi, K. Ikuina, N. Senba, N. Takahashi, and K. Ohata, "Low cost multi-layer ceramic package for a flip-chip MMIC up to W-band," IEEE MTT-S Int. Microwave Symposium Digest, pp. 57-60, Boston, MA, June 2000.

[12] Y. H. Suh, and K. Chang, " A new millimeter-wave printed dipole phased array antenna using microstrip-fed coplanar stripline Tee junctions," IEEE Transactions on Antennas and Propagation, vol. 52, no. 8, pp. 2019-2026, August 2004. 